\title{
Asymmetric Segregation of Numb in Retinal Development and the Influence of the Pigmented Epithelium
}

\author{
Michel Cayouette, ${ }^{1}$ Alan V. Whitmore, ${ }^{1}$ Glen Jeffery, ${ }^{2}$ and Martin Raff ${ }^{1}$ \\ ${ }^{1}$ Medical Research Council Laboratory for Molecular Cell Biology and the Biology Department, University College \\ London, London WC1E 6BT, United Kingdom, and 2Institute of Ophthalmology, University College London, London EC1V \\ 9EL, United Kingdom
}

Asymmetric segregation of cell-fate determinants during cytokinesis plays an important part in controlling cell-fate choice in invertebrates. During Drosophila neurogenesis, for example, asymmetric segregation of the Numb protein, which inhibits Notch signaling, is necessary for the two daughter cells of a division to have different fates. In vertebrates, the role of asymmetric segregation of cell-fate determinants is uncertain, and the way the process might be regulated is unknown. We have studied the orientation of cell divisions and the distribution of Numb in the developing rat retina. We show that, whereas most retinal neuroepithelial cells divide with their mitotic spindles oriented parallel to the plane of the neuroepithelium, a substantial minority divides with their spindles oriented perpendicularly. The proportion of these vertically dividing cells changes during development, peaking around the day of birth. Numb appears

How do individual neuroepithelial cells choose between alternative fates during development? In invertebrates, asymmetric cell divisions play an important part in cell-fate choice by differentially distributing cell-fate-determining proteins to the two daughter cells (Horvitz and Herskowitz, 1992; Rose and Kemphues, 1998; Lu et al., 2000). The unequal partitioning is achieved by first segregating the cell-fate determinants to one pole of the mother cell and then positioning the mitotic spindle in such a way that only one daughter cell receives the determinants when the mother cell divides.

In Drosophila, the differential segregation of the Numb protein to one of the two daughter cells of an asymmetric division is required for the daughters to adopt distinct fates during the development of the nervous system, muscle, and malpighian tubules (Uemura et al., 1989; Rhyu et al., 1994; Knoblich et al., 1995; Spana et al., 1995; Carmena et al., 1998; Wan et al., 2000). In neuroblasts, for example, Numb becomes localized to the cytosolic face of the basal plasma membrane during mitosis, and the mitotic spindle orients along the basal-apical axis, so that

Received March 9, 2001; revised May 1, 2001; accepted May 15, 2001.

This research was funded by a Human Frontier Science Program Long-Term Fellowship (M.C.), the Medical Research Council (A.V.W. and M.R.), and the Royal Society (A.V.W.). We are grateful to Yuh Nung Jan for the Numb antibodies, Spyros Artavanis-Tsakonas for the Notch-1 antibodies, Juergen Knoblich for insightful comments on this manuscript, Julian Lewis and Mark Eddison for useful discussions, John Dawson for helpful discussion and advice on polar coordinate systems, and members of the Raff laboratory for stimulating discussion and support.

M.C. and A.V.W. contributed equally to this work.

Correspondence should be addressed to Michel Cayouette and Alan Whitmore, Medical Research Council Laboratory for Molecular Cell Biology, University College London, Gower Street, London, WC1E 6BT, UK. E-mail: m.cayouette@ ucl.ac.uk and a.whitmore@ucl.ac.uk.

Copyright (C) 2001 Society for Neuroscience 0270-6474/01/215643-09\$15.00/0 to be inherited only by the apical daughter cell when a neuroepithelial cell divides vertically. Similarly, in dissociated cell cultures, some retinal neuroepithelial cells divide asymmetrically and distribute Numb to only one of the two daughter cells, suggesting that the dissociated cells can retain their polarity in vitro. Using retinal explant cultures, we find that the retinal pigment epithelium apparently promotes vertical divisions in the neural retina. To our knowledge, this is the first evidence that asymmetric segregation of cell-fate determinants may contribute to cell diversification in the mammalian retina and that an epithelium controls this process by influencing the plane of division in the adjacent neural retina.

Key words: retina; CNS; development; neuroepithelial cells; Numb; asymmetric division; mitotic spindle

only the basal daughter cell inherits Numb (Rhyu et al., 1994). Numb is thought to influence cell fate, at least in part, by inhibiting Notch signaling (Frise et al., 1996; Guo et al., 1996; Spana and Doe, 1996).

It has long been speculated that asymmetric divisions might also play a part in cell-fate choice in the developing vertebrate nervous system. The first direct evidence that asymmetric divisions could produce daughter cells that have different fates came from time-lapse imaging studies of neural precursors in explanted slices of developing ferret telencephalon in which cleavage orientation was found to be correlated with the behavior of the two daughter cells (Chenn and McConnell, 1995). In the same study, it was found that Notch1, a mammalian homolog of Drosophila Notch, was concentrated basally in neuroepithelial cells and was therefore presumably inherited only by the basal daughter of vertical divisions. Further evidence that asymmetric segregation of cell-fate determinants may have a role in cell-fate choice in vertebrate neurogenesis has come from studies of the distribution of Numb homologs in mammals and chicks (Verdi et al., 1996; Zhong et al., 1996; Wakamatsu et al., 1999). It remains to be directly demonstrated, however, that Numb influences cell-fate choice in vertebrates, as it clearly does in flies.

The retina is an attractive part of the vertebrate CNS in which to address the question of how cells choose between alternative fates. It is discrete and readily accessible, and the different cell types are produced in a well defined chronological order; each cell type has a characteristic morphology and location that makes it relatively easy to identify. Although there is evidence that both cell-intrinsic factors and cell-cell interactions contribute to cellfate decisions in the retina (Cepko et al., 1996; Harris, 1997; 
Cepko, 1999; Livesey and Cepko, 2001), their relative importance and molecular bases remain largely unknown. The possible role of asymmetric segregation of cell-fate determinants in these decisions has not been explored.

We show here that some dividing rat retinal neuroepithelial cells have their mitotic spindles oriented $45-90^{\circ}$ away from the plane of the neuroepithelium. The proportion of these vertically dividing cells depends on the underlying pigment epithelium and changes during development. The Numb protein is apically distributed in retinal neuroepithelial cells and seems to be inherited by only the apical daughter cell of a vertical cell division. This is the first evidence that asymmetric segregation of cell-fate determinants may contribute to cell diversification in the mammalian retina.

\section{MATERIALS AND METHODS}

Histology and immunohistochemistry. We studied the orientation of mitotic spindles and the intracellular distribution of cell-fate determinants in the retinas of Lister hooded rats. Animals were killed at different ages during development, and the eyes were removed and fixed in $2 \%$ paraformaldehyde overnight at $4^{\circ} \mathrm{C}$. Then, the eyes were cryoprotected in $20 \%$ sucrose, embedded in a 2:1 mixture of $20 \%$ sucrose/OCT, and rapidly frozen in liquid nitrogen. Cryosections were cut at $16-20 \mu \mathrm{m}$ and mounted on polylysine-coated slides (Menzel-Glaser, Braunscheig, Germany). All reagents were from Sigma (Poole, UK) unless otherwise stated.

To ensure uniformity in the analysis, we selected sections passing through the optic nerve head. We then labeled the centrosomes to help determine the orientation of mitotic spindles. We post-fixed retinal sections in $70 \%$ ethanol for $10 \mathrm{~min}$ at $-20^{\circ} \mathrm{C}$, blocked them in $10 \%$ goat serum in $1 \%$ Triton X-100 in PBS (1\% Triton), and incubated them overnight at room temperature in rabbit anti- $\gamma$-tubulin antibodies (diluted $1: 500$ in $1 \%$ Triton plus $5 \%$ goat serum). Bound antibodies were detected by incubation for $2 \mathrm{hr}$ with biotinylated goat anti-rabbit Ig antibodies, followed by a $1 \mathrm{hr}$ incubation in Streptavidin-FITC (diluted 1:100 in PBS; both from Amersham Pharmacia Biotech, Braunschweig, Germany). For both Numb and Notch-1 staining, retinal sections or dissociated retinal cells were blocked in $10 \%$ goat serum in $0.1 \%$ Triton and incubated overnight at $4{ }^{\circ} \mathrm{C}$ with one of the following antibodies: a monoclonal anti-Numb antibody made against amino acids 176-291 (diluted 1:500 in $0.1 \%$ Triton plus 5\% goat serum; Becton Dickinson, Oxfordshire, UK), affinity-purified rabbit anti-m-Numb antibodies (diluted 1:500; from Y.-N. Jan, University of California at San Francisco, San Francisco, CA), a rat monoclonal anti-Notch-1 antibody (diluted 1:250; from S. Artavanis-Tsakonas, Massachusetts General Hospital Cancer Center), or affinity-purified rabbit anti-Notch-1 antibodies (diluted 1:250; Santa Cruz Biotechnology, Santa Cruz, CA). Bound anti- bodies were detected using biotinylated goat anti-rat, anti-rabbit, or anti-mouse Ig antibodies (all 1:100 in PBS), followed by StreptavidinFITC as described above. In all cases, control sections and cells without primary antibodies showed no cellular staining.

Immunostained sections were counterstained with propidium iodide (5 $\mu \mathrm{g} / \mathrm{ml}$ in PBS plus $25 \mathrm{U}$ of RNase A) to visualize nuclear morphology. The sections were examined with a laser scanning confocal microscope (MRC 600 and 1024; Bio-Rad, Hercules, CA), and images were merged and pseudocolored using Bio-Rad Confocal Assistant, Adobe Photoshop, or Analyze PC.

In some experiments, dissociated retinal cells were triple labeled. They were first labeled with the affinity-purified rabbit anti-Notch-1 antibodies that were detected as above. Then, they were labeled with the monoclonal anti-m-Numb antibody, which was detected with Texas-redconjugated goat anti-mouse Ig (diluted 1:100 in PBS). Finally, the cells were stained with bisbenzimide (Hoescht 33342) to visualize all nuclei.

Retinal explant and dissociated cell cultures. Neonatal [postnatal day 0 (P0)] eyes were enucleated and transferred to HBSS. To culture retinal explants with the retinal pigment epithelium (RPE) in place, we incubated the eyes in $2.5 \%$ dispase II in HBSS for $45 \mathrm{~min}$ at $37^{\circ} \mathrm{C}$ and then dissected the retina with care to leave the RPE attached. Treatment with dispase was essential to leave the RPE attached to the neural retina consistently. To culture neural retina without the RPE, the eyes were incubated in the same conditions as for the explants with RPE but without the dispase, and the neural retina was dissected free from the RPE in HBSS. The explants were cut into small pieces $\left(\sim 3 \mathrm{~mm}^{2}\right)$ and transferred onto a Millicell CM organotypic culture insert (Millipore, Bedford, MA) that was floating in $1.2 \mathrm{ml}$ of a 50:50 mixture of DMEM and F12 (DMEM-F12) containing 5\% FCS and penicillin-streptomycin. Explants were cultured for $24 \mathrm{hr}$ at $37^{\circ} \mathrm{C}$ in an $8 \% \mathrm{CO}_{2}$ atmosphere and fixed and immunostained as described above.

Dissociated retinal cell cultures were performed as previously described (Jensen and Raff, 1997) with the following changes. Retinas were dissected in HBSS and dissociated with $0.025 \%$ trypsin in HBSS for 7-10 min at $37^{\circ} \mathrm{C}$. Trypsin was inhibited with $20 \%$ FCS in DMEM-F12 medium containing $0.04 \%$ DNase. Cells were dissociated by trituration in the inhibitor solution, centrifuged, and resuspended in DMEM-F12 medium. The cells were plated then in serum-free medium consisting of a 1:1 mixture of DMEM-F12 with N2 supplement/Neurobasal with B27 supplement, to which basic FGF $(10 \mathrm{ng} / \mathrm{ml})$, epidermal growth factor (EGF) (100 ng/ml), 8-(4-chlorophenylthio)adenosine 3':5'-cyclic monophosphate $(0.1 \mathrm{~mm}), 3$-isobutyl-1-methylxanthine $(0.1 \mathrm{mM})$, and penicillin-streptomycin was added. Cells were cultured at $37^{\circ} \mathrm{C}$ in a humidified incubator in $8 \% \mathrm{CO}_{2}$, either in $25 \mathrm{~cm}$ T-flasks (Falcon; 10,000 cells) or on $13 \mathrm{~mm}$ glass coverslips (1,000 cells), both coated with poly-D-lysine (10 $\mu \mathrm{g} / \mathrm{ml})$ and laminin $(10 \mu \mathrm{g} / \mathrm{ml})$.

Quantification of mitotic spindle orientation. Previous studies of spindle orientation in other tissues have been criticized because of the difficulty of accurately assessing the orientation of cells within tissues. We therefore have gone to some trouble to develop a robust, objective, and

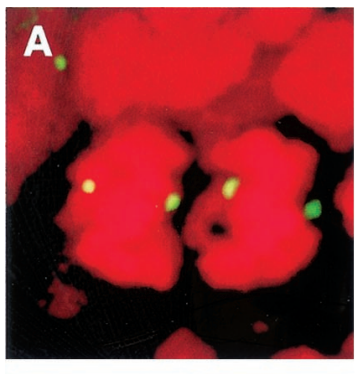

Figure 1. Mitotic spindle orientations in dividing retinal neuroepithelial cells. P0 retinal sections were stained with both anti- $\gamma$-tubulin antibodies to visualize the centrosomes (green) and propidium iodide to visualize DNA (red). Both metaphase $(A-C)$ and anaphase-telophase $(D-F)$ mitotic cells were analyzed. Most dividing cells had the mitotic spindle aligned horizontally relative to the plane of the tissue $(A, D)$, but in some cells the spindle was aligned perpendicular to the plane of the tissue $(C, F)$ or in an intermediate orientation $(B, E)$. The retinal pigment epithelium is down in all figures.

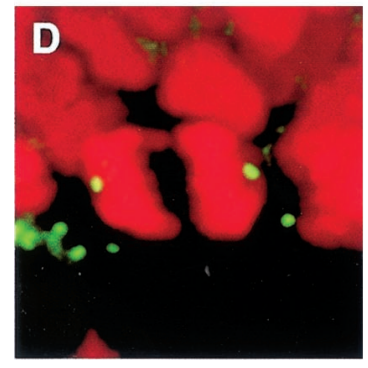

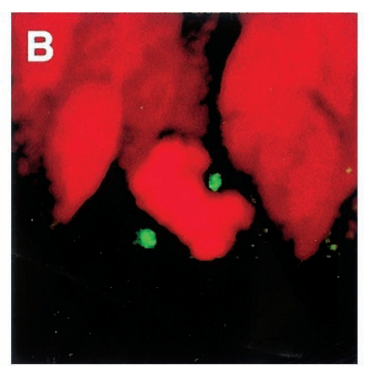
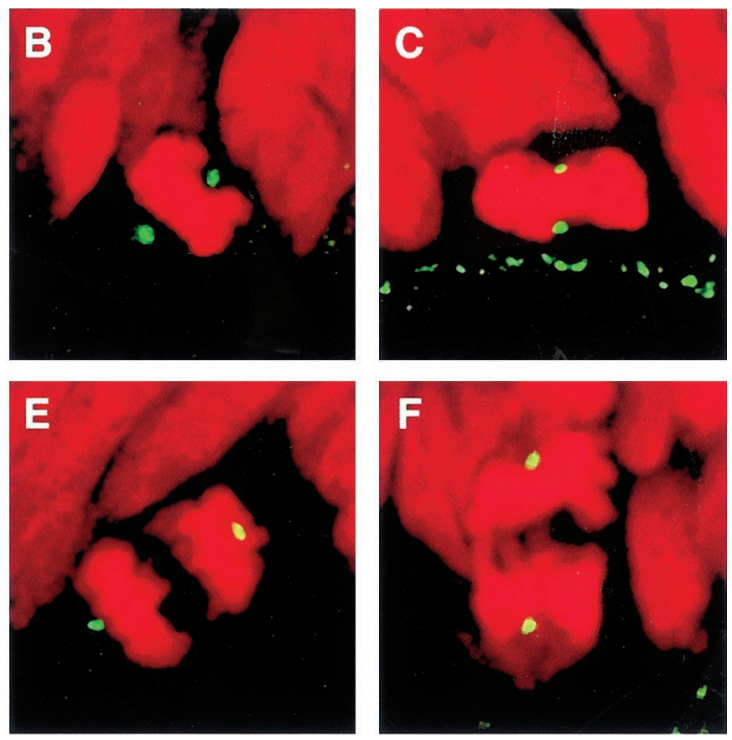
reproducible method of analysis (see Appendix). Serial optical sections of a region of interest, selected at random, were captured with a confocal microscope at $0.20 \mu \mathrm{m}$ intervals and stored on a computer. All sampled volumes were in the range $\sim 150 \times 100 \times 10-20 \mu \mathrm{m}$ and contained between 0 and 10 mitotic cells. Every cell with paired centrosomes was analyzed, irrespective of the mitotic stage. If there was any doubt that the chromosomes and two centrosomes belonged to the same spindle, the cells were reconstructed in three dimensions from the optical sections and rotated at will using AnalyzePC (Mayo Clinic, Rochester, MN). This reconstruction was particularly helpful for assessing cells that were dividing "end on" to the plane of section. Using three-dimensional coordinate geometry, we calculated the orientation of the spindles from the $x, y$, and $z$ coordinates of the centrosome pairs (see Fig. 7 in Appendix). All spindle angles were defined with reference to the plane corresponding to the interface between the RPE and the neural retina. All computations were performed using a custom-written MATLAB program (The Mathworks Inc., Natick, MA). Frequency histograms of the population distribution of spindle angles were plotted and compared between experimental conditions using various statistical tests, as indicated in the figure legends.

\section{RESULTS}

\section{Spindle orientations in dividing retinal neuroepithelial cells}

To examine spindle orientations in dividing rat retinal neuroepithelial cells during development, we stained fixed retinal sections with anti- $\gamma$-tubulin antibodies to label the centrosomes and propidium iodide to label the DNA. Using confocal microscopy, we identified the centrosome pairs in cells in either metaphase (Fig. $1 A-C$ ) or anaphase-telophase (Fig. $1 D-F$ ) and used threedimensional coordinate geometry to measure the angle of the spindle axis relative to the plane of the neuroepithelium (see Materials and Methods and Appendix). In newborn animals (P0), most retinal neuroepithelial cells divided with their spindles aligned roughly parallel to the tissue plane (Fig. $1 A-D$ ). We observed some cells, however, dividing with their spindles rotated up to $90^{\circ}$ relative to the tissue plane (Fig. $1 B, C, E, F$ ), raising the possibility that these cells may have altered the orientation of their spindle to segregate cell-fate determinants asymmetrically to their two daughter cells.

\section{Changes in the proportion of vertical spindles with developmental age}

Because the different cell types in the retina are generated in a predictable chronological order (Young, 1985), if asymmetric divisions are important in generating specific retinal cell types, one might expect a correlation between the proportion of vertically dividing cells and the production of those cell types. For simplicity, we used an arbitrary spindle angle of $<45^{\circ}$ relative to the tissue plane to classify a division as "horizontal" and $\geq 45^{\circ}$ to classify a division as "vertical." As shown in Figure $2 A$, at embryonic day 18 (E18), only $\sim 3 \%$ of dividing cells had their spindles oriented vertically. In contrast, at $\mathrm{P} 0,>20 \%$ of dividing cells had vertically oriented spindles (Fig. $2 B$ ), and at P4, the proportion decreased to $10 \%$ (Fig. $2 C$ ).

Is it possible that the vertical spindles reorient to a horizontal position before the cell divides? In Drosophila, spindle reorientation has been shown to occur at metaphase (Kaltschmidt et al., 2000). We therefore specifically analyzed spindle orientation in anaphase-telophase cells, which are unlikely to reorient their spindles before division. We found that $25 \%$ of the spindles were oriented $>45^{\circ}$ away from the horizontal in the $\mathrm{P} 0$ retina.

Taken together, these results support the conclusion that a proportion of retinal neuroepithelial cells divides vertically and that this proportion changes during retinal development.
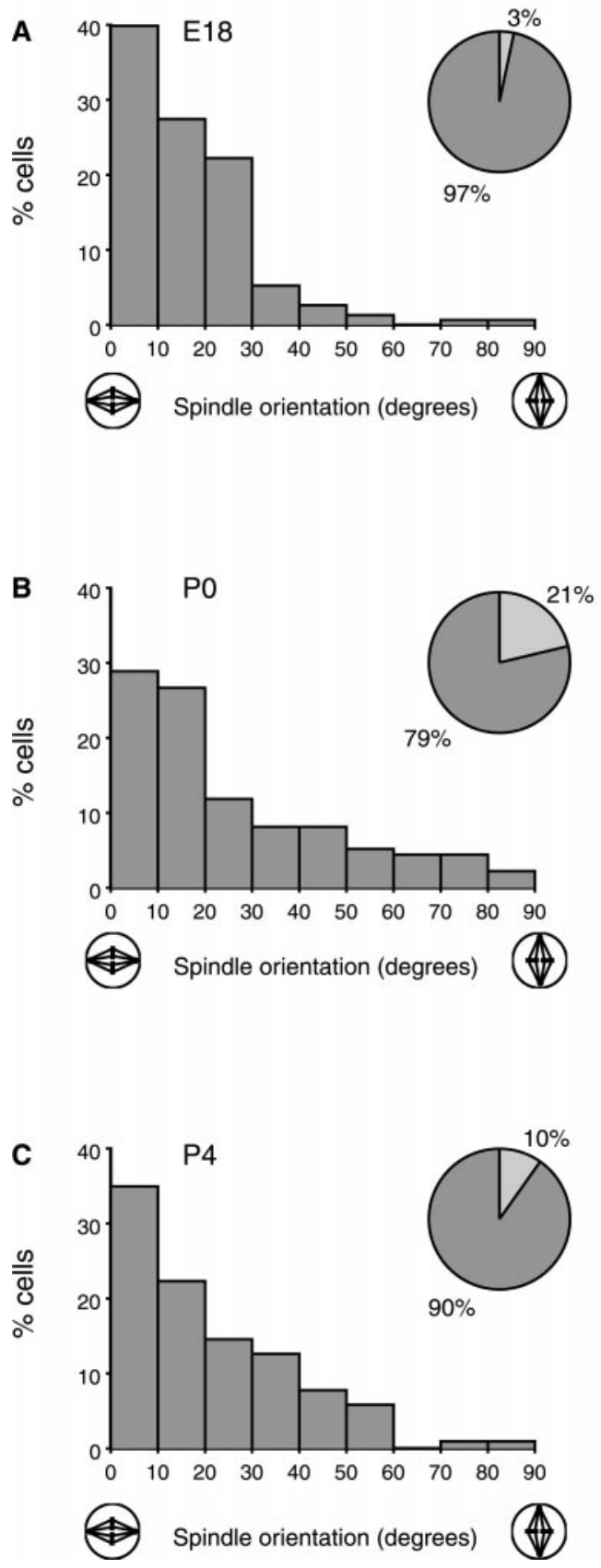

Figure 2. The distribution of mitotic spindle orientations at different developmental ages. The graphs show frequency histograms of the orientations of mitotic spindles in embryonic and postnatal rat retinas at E18 $(A), \mathrm{P} 0(B)$, and $\mathrm{P} 4(C)$. Four animals were analyzed at E18 $(n=153$ cells) and P0 ( $n=135$ cells), whereas three animals were analyzed at P4 ( $n=104$ cells $)$. The distributions at different times were compared using the Kolmogorov-Smirnov test and found to be significantly different for embryonic and postnatal retina: E18 versus $\mathrm{P} 0, p=0.0001$; E18 versus P4, $p=0.005$. The pie charts show the proportions of vertical (light gray) and horizontal (dark gray) spindles at the different times, where vertical was defined as an angle $\geq 45$ degrees. The differences in the proportions are highly significant when compared using the $\chi^{2}$ test: E18 versus $\mathrm{P} 0, p<$ 0.0001 ; E18 versus P4, $p=0.03$.

\section{Influence of RPE on spindle orientation in the neuroepithelium}

The RPE is essential for the development and survival of the neural retina; when it is selectively ablated, the retina and, in 
A +RPE

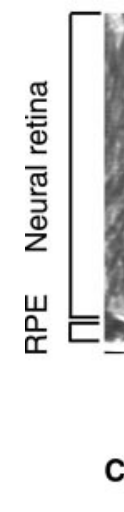

Figure 3. The effect of the retinal pigment epithelium (RPE) on the orientation of mitotic spindles. $A, B$, Cryostat sections through $\mathrm{P} 0$ retinal explants cultured for $24 \mathrm{hr}$ either with $(A)$ or without $(B)$ the RPE in place. $C, D$, Frequency histograms of the orientations of mitotic spindles in comparable retinal explants cultured for $24 \mathrm{hr}$ either with $(C)$ or without $(D)$ the RPE. The results were pooled from two separate experiments that involved a total of 11 rats $(+R P E$ explants, $n=193$ cells; $-R P E$ explants, $n=176$ cells). Pie charts show the proportion of vertical (light gray) and horizontal (dark gray) spindles in each condition. The Kolmogorov-Smirnov test shows that the difference between the frequency distributions in $A$ and $B$ is highly significant $(p=0.0088)$. Comparison of the proportions of vertical and horizontal spindles using the $\chi^{2}$ test also shows a significant difference $(p=0.0342)$.

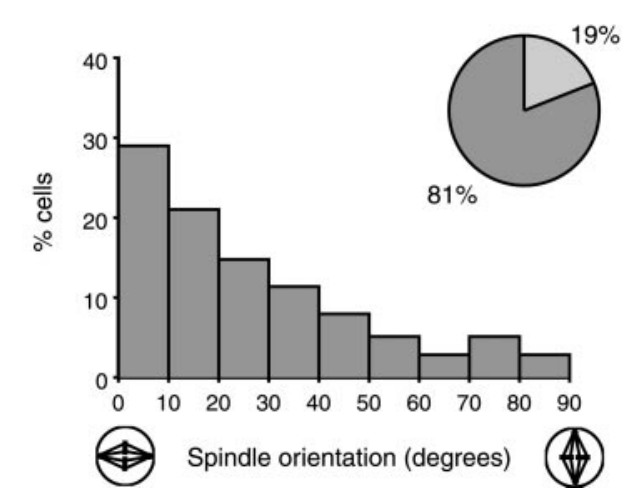

B -RPE

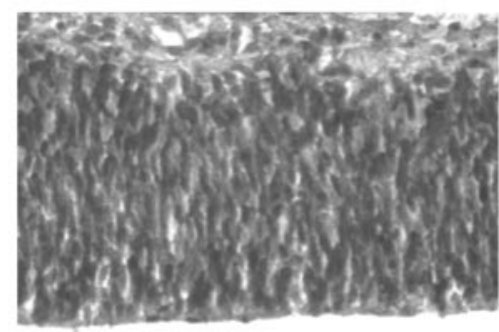

D

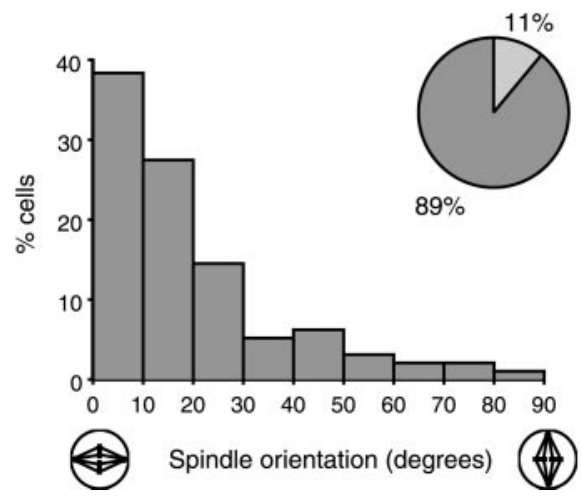

some conditions, the entire eye fail to develop (Raymond and Jackson, 1995). To determine whether the RPE influences spindle orientation in the neural retina, we studied explants of retina, with or without the RPE, after $24 \mathrm{hr}$ in culture (Fig. $3 A, B$ ).

As shown in Figure 3, there was a significant difference between the proportion of vertical spindles with and without the RPE. With the RPE, the proportion of vertical spindles $(\sim 20 \%)$ was not significantly different from that seen in the $\mathrm{P} 0$ retina in vivo (compare Figs. $2 B, 3 C$ ), suggesting that the enzyme treatment required to prepare retinal explants with the RPE in place did not affect the normal distribution of spindle orientations. When the explants were cultured without the RPE, the proportion of vertical spindles was significantly reduced to about half of that seen in either the explants with the RPE (Fig. 3D) or in vivo at the comparable age (Fig. 2B).

\section{Asymmetric segregation of Numb in vertical divisions}

The presence of vertical mitotic spindles in some retinal neuroepithelial cells raised the possibility that these cells divide asymmetrically and distribute cell-fate determinants unequally to their two daughter cells at cytokinesis. To test this possibility, we examined the distribution of the cell-fate determining protein Numb in retinal neuroepithelial cells.

Retinal sections from $\mathrm{P} 0$ rats were stained using a monoclonal anti-Numb antibody. The sections were also stained with propidium iodide and were analyzed using a confocal microscope. As expected, Numb was mostly distributed at the periphery of retinal neuroepithelial cells and was highly concentrated at the apical side of the cells (Fig. 4A), where it was mainly associated with fine, finger-like structures (Fig. $4 B$ ). Because these structures were not seen in mitotic cells (Fig. $4 C-F$ ), they probably represent the apical membrane of neuroepithelial cells in interphase.

In mitotic neuroepithelial cells, Numb staining was also consis- tently concentrated at the apical pole, with little or no staining at the basal pole (Fig. $4 C-F$ ). This asymmetric distribution was observed regardless of the orientation of the mitotic spindle. As shown in Figure 4, $C$ and $D$, cells dividing with their spindle aligned parallel to the plane of the neuroepithelium are likely to distribute Numb symmetrically to both daughter cells. In contrast, cells dividing with their spindles oriented vertical to the neuroepithelium seemed to distribute Numb preferentially to the apical daughter (Fig. 4E,F). These results suggest that the more vertical a cell division is, the more likely it is to segregate Numb preferentially to the apical daughter cell.

Because the RPE influenced spindle orientation in explanted retinas, we investigated whether it also influenced the localization of Numb. We cultured explants for up to $72 \mathrm{hr}$ without RPE and were unable to detect any change in the distribution of Numb labeling, which was always located mainly at the apical pole of neuroepithelial cells, whether the cells were in mitosis or not (data not shown).

\section{Asymmetric segregation of Numb in dissociated retinal cell cultures}

To confirm our impression that Numb can be asymmetrically segregated between the two daughter cells of a neuroepithelial cell division, we dissociated $\mathrm{P} 0$ retinas and cultured the cells at low density in serum-free medium in the presence of basic FGF and EGF. In these conditions, some neuroepithelial cells continued to divide. After 3-4 hr, any cells that were in clusters of two or more were ringed and excluded from the analysis. After 24-28 $\mathrm{hr}$, the cells were fixed and stained for Numb. We observed many cell doublets in which the two cells seemed either to be in the process of cytokinesis or to have recently completed cytokinesis. The proportion of cell doublets increased from $6 \%$ at 3-4 hr to 

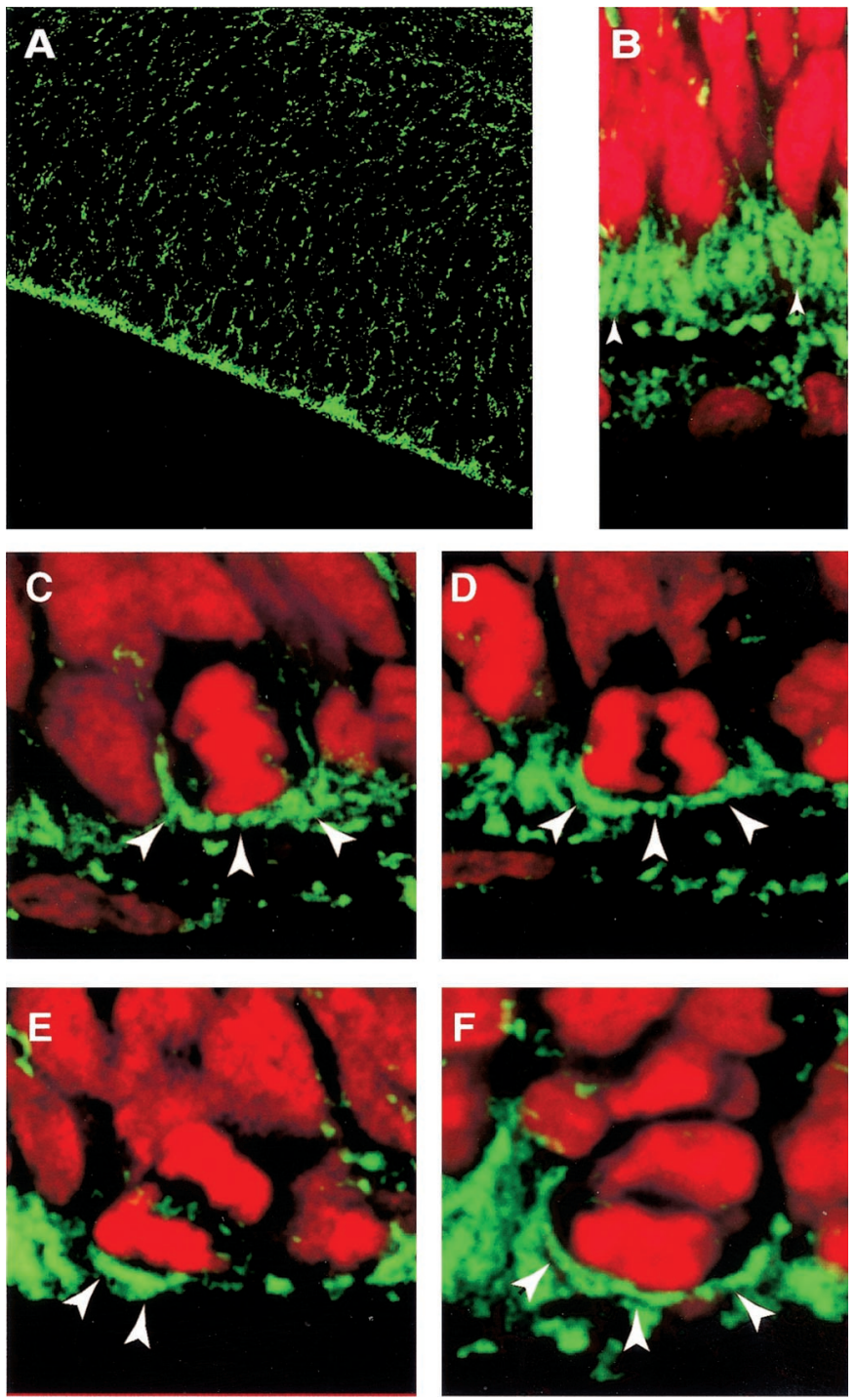

Figure 4. Apical localization of Numb in $\mathrm{P} 0$ retinal neuroepithelial cells. Retinal sections were stained with monoclonal anti-Numb antibody (green) and propidium iodide (red). A, Low magnification view showing that Numb staining is mainly concentrated on the apical side of the neuroepithelium. $B$, High magnification view showing the finger-like staining of Numb associated with the apical pole of interphase neuroepithelial cells (arrowheads point to two examples of finger-like staining). $C-F$, Numb distribution in mitotic neuroepithelial cells (arrowheads). Metaphase $(C)$ and anaphase-telophase $(D)$ cells dividing with their spindles aligned horizontally, relative to the plane of the tissue, show an apical crescent of Numb, suggesting that both daughter cells of these divisions will inherit Numb. In cells dividing with their mitotic spindles aligned more perpendicularly to the plane of the tissue ( $E$ and $F$ show two examples of cells in anaphase-telophase), Numb is likely to be inherited preferentially by the apical daughter cell.

$24 \%$ at $24-28 \mathrm{hr}$, suggesting that a significant proportion of single cells divided during the culture period.

In many single cells, Numb staining was confined to one side of the cell (Fig. 5A, $a-c$ ), whereas in others it was diffusely distributed around the cell periphery (data not shown). In most cell doublets, Numb staining was found in both cells (Fig. $5 A, d-f$ ), which were probably produced by a symmetric division during the culture period; the staining was usually polarized and always at the same side of the two cells (Fig. $5 A, d-f$ ). In some doublets, however, only one of the two cells stained for Numb, and the staining was confined to one pole of the cell, which was usually the pole opposite to the cell-cell contact zone (Fig. 5A, g- $i$ ), consistent with the cell being produced by an asymmetric division during the culture period. Thus, some dissociated retinal neuroepithelial cells can retain their polarity in vitro and apparently segregate Numb asymmetrically to their daughter cells when they divide.

Because Numb inhibits Notch signaling (Frise et al., 1996; Guo et al., 1996; Spana and Doe, 1996; Wakamatsu et al., 1999) and Notch has been reported to be asymmetrically segregated to the basal daughter cell of vertical divisions in the developing ferret cortex (Chenn and McConnell, 1995), we double-labeled dissociated retinal cells for Notch-1 and Numb. Irrespective of the distribution of Numb, Notch-1 was always diff usely distributed on the cell surface (Fig. 5B). Thus, Notch-1 is apparently always distributed to both daughter cells during cytokinesis, at least in cultured retinal cells.

\section{DISCUSSION}

In examining propidium-iodide-stained sections of the developing retina, it is difficult to be certain of the orientation of a mitotic spindle. We have circumvented this problem by using confocal microscopy and three-dimensional coordinate geometry to determine spindle orientation. Our results indicate that some dividing neuroepithelial cells in the developing rat retina have their mitotic spindle oriented $45-90^{\circ}$ away from the plane of the neuroepithelium and seem to distribute Numb preferentially to the apical daughter cell.

\section{Asymmetric segregation of Numb}

Numb plays a crucial part in cell-fate determination during Drosophila development, and there is some indirect evidence that it may also do so in vertebrates. Two mammalian homologs of Numb have been identified in mouse, both of which are expressed in the nervous system: Numblike is cytosolic in postmitotic neurons of the cortical plate (Zhong et al., 1997), whereas m-Numb is bound to the cytosolic face of the plasma membrane at the apical pole of mitotic cortical neuroepithelial cells (Zhong et al., 1996, 1997). Mice homozygous for a loss-of-function mutant allele of $m$-numb exhibit severe defects in cranial neural tube closure and precocious neuron production in the forebrain, and they die at approximately E11.5, suggesting a role for Numb in maintaining a progenitor cell character during cortical neurogenesis (Zhong et al., 2000).

On the basis of its apical distribution in mouse cortical neuroepithelial cells, Zhong et al. (1996) suggested that m-Numb would be distributed into either one or both daughter cells during cell division, depending on the orientation of the cell division. Our findings show that this is the case in the neonatal rat retina. We observed that Numb is asymmetrically distributed in both mitotic and interphase cells, suggesting that it is not degraded or redistributed in the cell after mitosis but remains apical at all stages of the cell cycle. Consequently, in mitotic cells with a horizontally oriented spindle, Numb seems to be present in about equal amounts in both of the nascent daughter cells, whereas in cells with a vertically oriented spindle it is preferentially segregated to the nascent apical daughter cell, with very little, if any, in the nascent basal cell. Even in low-density dissociated retinal cell cultures, Numb is asymmetrically localized in some cells and seems to be preferentially distributed to one of the two daughter cells when some retinal neuroepithelial cells divide. These results 
A

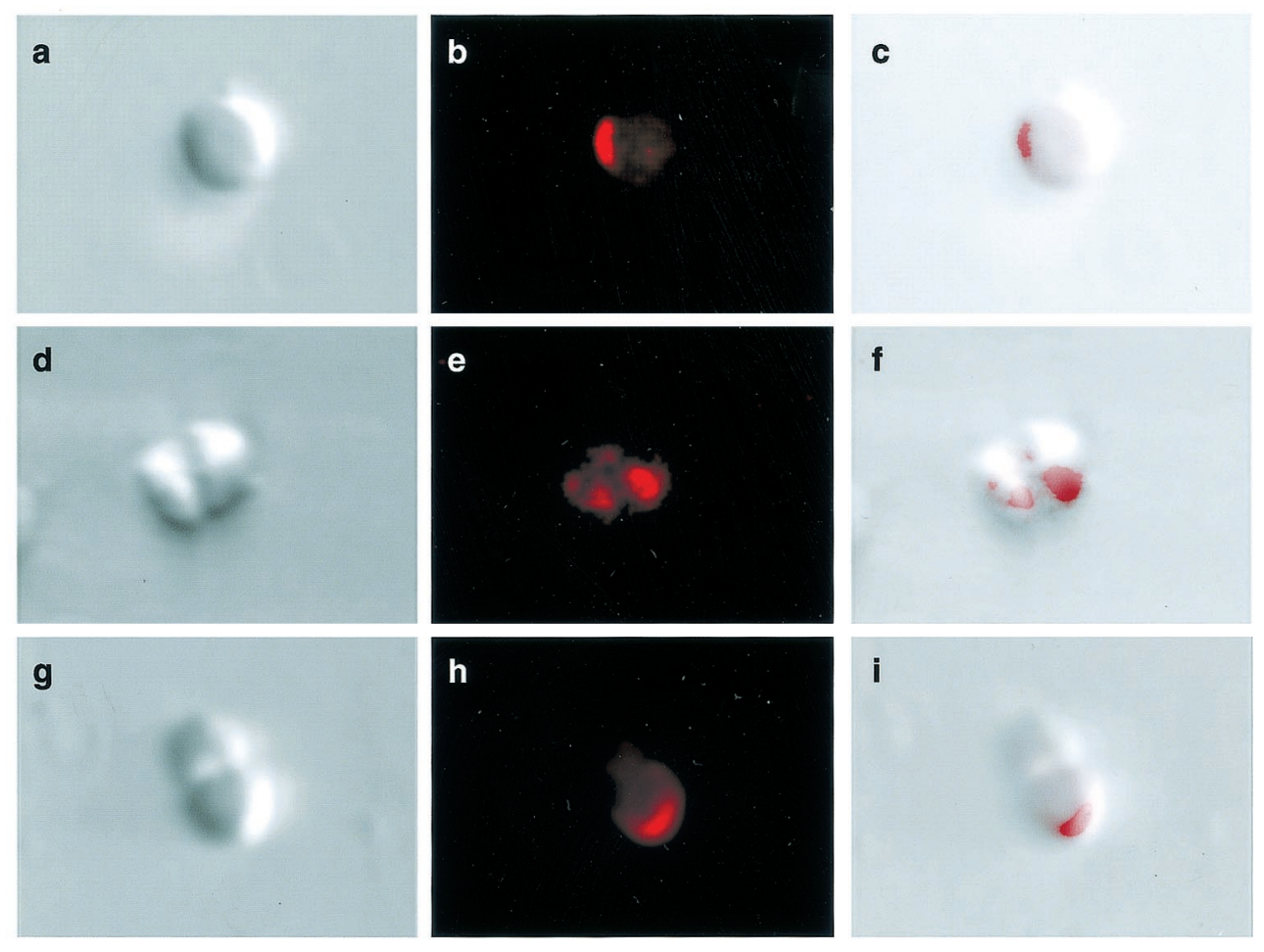

B
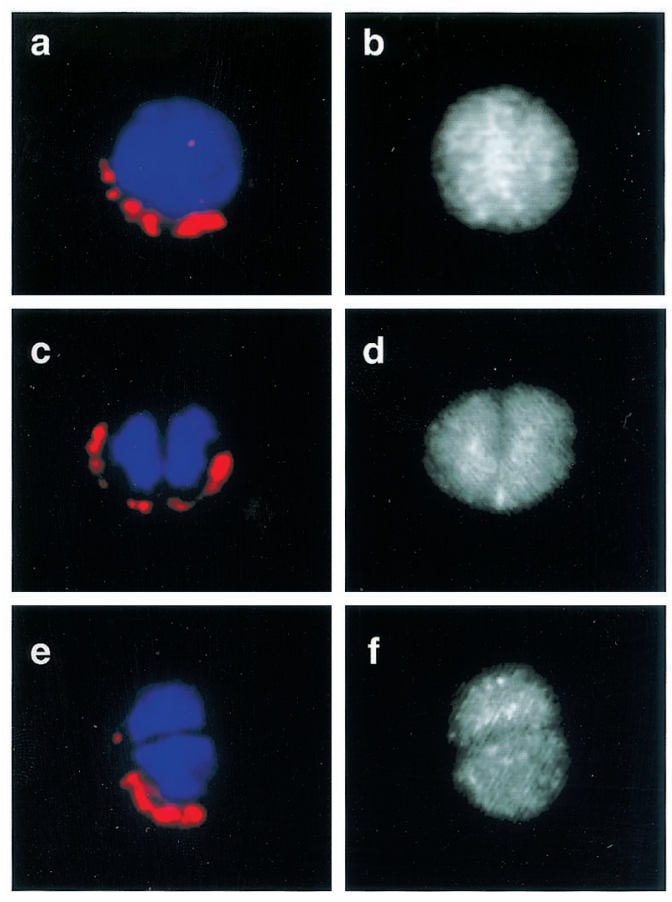

Figure 5. Asymmetric distribution of Numb and symmetric distribution of Notch-1 in dissociated retinal cell cultures. $A$, Differential-interference contrast images of dissociated retinal cells $(a, d, g)$, immunofluorescence images of Numb staining (red) of the corresponding cells $(b, e, h)$, and overlaid images of both $(c, f, i)$. Some single cells show Numb confined to one pole $(a-c)$. In most cell doublets, Numb is found at the same pole in both cells, consistent with the cells being produced by a symmetric division in culture $(d-f)$. In some doublets, however, Numb is found only in one of the cells, with a distribution consistent with the two cells being produced by an asymmetric division in which only one daughter cell inherited Numb $(g-i)$. $B$, Numb $(a, c, e)$ and Notch $(b, d, f)$ immunofluorescence staining in dissociated retinal cells. The DNA is stained in blue with Hoescht dye in $a, c$, and $e$. In a single cell in which Numb is segregated to one pole $(a)$, Notch-1 is evenly distributed on the cell surface $(b)$. In cell doublets $(c-f)$, Notch-1 is always observed evenly distributed on the surface of both cells $(d, f)$, irrespective of whether Numb is found on both cells $(c)$ or only on one $(e)$.

suggest that polarity can be maintained in dissociated cells in culture and support the conclusion that some retinal neuroepithelial cells in vivo divide asymmetrically and distribute Numb to only one daughter cell. Because Drosophila Numb is necessary for the daughter cells of asymmetric divisions in the fly to adopt distinct fates (Rhyu et al., 1994; Spana et al., 1995) and m-Numb can rescue the Numb-deficient fly mutant (Zhong et al., 1996), it seems likely that the asymmetric segregation of Numb plays a part in cell-fate choice during mammalian retinal development.

\section{The possible role of Numb}

Using time-lapse microscopy of dividing cells in slices of developing ferret cerebral cortex, Chenn and McConnell (1995) reported that the orientation of cell division could influence the 
Interphase M-phase

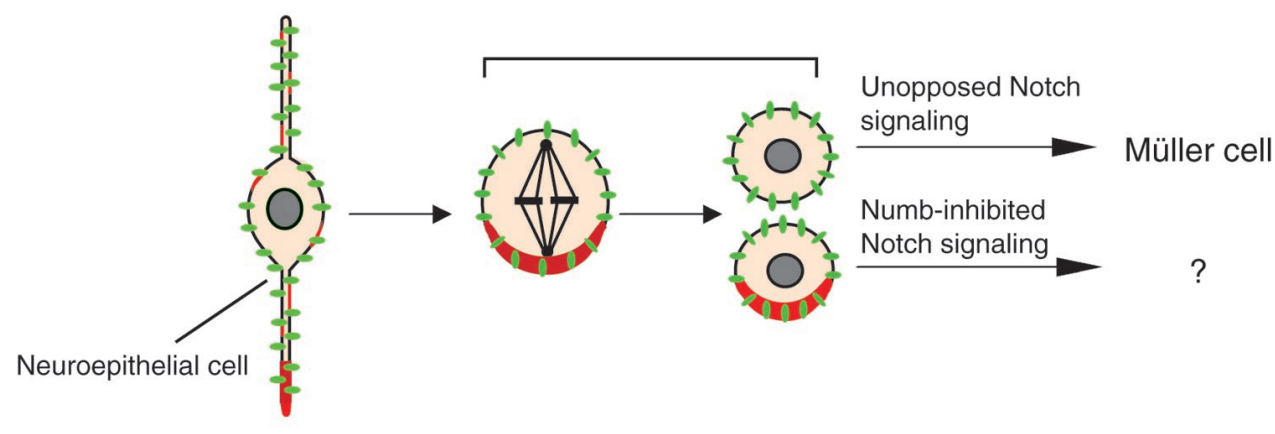

Numb

Notch-1
Figure 6. A model for how asymmetric cell division in the retina may influence cell-fate. Some retinal neuroepithelial cells reorient their mitotic spindles and divide vertically. Because Numb is concentrated at the apical pole of the cells, it is likely to be inherited preferentially by the apical daughter cell of such divisions, where it inhibits Notch signaling. The basal daughter cell inherits little Numb, allowing unopposed Notch signaling and differentiation into a Müller cell. The apical cell might divide again or develop into another cell type. fates of daughter cells. Horizontal divisions produced behaviorally and morphologically identical daughters that resembled precursor cells. In contrast, vertical divisions produced basal daughters that behaved like young migratory neuroblasts and apical daughters that remained within the proliferative zone. Putting these findings together with the results of Zhong et al. (1996) on the localization of $\mathrm{m}-\mathrm{Numb}$ in the proliferative zone of the developing mouse cerebral cortex, it seems likely that the apical daughter cell that inherits m-Numb in vertical divisions would remain a neuroepithelial cell. In view of the well known role of Notch in lateral inhibition (for review, see Lewis, 1998), this conclusion may seem surprising, because cells that inherit Numb would be expected to have reduced Notch signaling (Frise et al., 1996; Guo et al., 1996; Spana and Doe, 1996). It is now clear, however, that Notch signaling can have diverse roles in development and can sometimes promote differentiation instead of inhibiting it (for review, see Wang and Barres, 2000).

Our results show that the proportion of dividing retinal neuroepithelial cells with vertical spindles varies with developmental age. Few are observed at E18 (and at E14 or E16; data not shown), whereas many are observed at birth, and intermediate proportions are observed at $\mathrm{P} 4$. This timing might reflect the normal time course of retinal cell production. Different cell types are born within specific, but overlapping, time windows in the developing mammalian retina. In rodents, for example, retinal ganglion cells and cones are produced mainly prenatally, whereas Müller and bipolar cells develop postnatally (Young, 1985). Rod photoreceptors, which make up $>70 \%$ of the cells in the adult rodent retina, are born over a prolonged period, spanning embryonic and postnatal development, but most are born around birth (Young, 1985). Thus, the high proportion of vertical divisions at birth could reflect the production of rods, Müller cells, or bipolar neurons (or a combination of these) around this time.

Recently, Notch signaling has been found to promote the development of glial cells, including Schwann cells in the peripheral nervous system (Morrison et al., 2000) and radial glial cells (Gaiano et al., 2000) and Müller cells (Furukawa et al., 2000) in the CNS. In the retina, overexpression of Notch-1 in retinal precursor cells using a replication-incompetent retroviral vector resulted in bigger clones that contained an abnormally large number of cells expressing a Müller cell marker (Furukawa et al., 2000). Because Numb inhibits Notch signaling and Notch seems to be inherited symmetrically by both daughter cells of all retinal neuroepithelial cell divisions, at least in culture (Fig. 5B), the apical daughter cells of vertical divisions in the retina might be inhibited from developing into Müller cells as a consequence of inheriting Numb. A basal cell, which receives Notch but not Numb, by contrast, might be encouraged to differentiate into a Müller cell as a result of unopposed Notch signaling (Fig. 6). Lineage analyses in vivo, after retroviral infection of the P0 rat retina, have found that clones never contain more than one Müller cell (Turner and Cepko, 1987), consistent with a model in which Müller cells develop exclusively from Numb-deficient basal daughter cells produced by asymmetric cell divisions. Even at birth, however, the great majority of divisions are horizontal, suggesting that symmetric divisions produce most cells born postnatally, where Notch signaling is at least partly counteracted by Numb.

\section{The role of the $\mathrm{RPE}$}

We find that the RPE can influence the orientation of the mitotic spindle in dividing cells in the adjacent retinal neuroepithelium, at least in explant culture. With the RPE still attached to a neonatal retinal explant, the proportion of vertical spindles is similar to that in the neonatal retina in vivo, suggesting that the dispase treatment used to prepare retinal explants with the RPE attached does not change the normal distribution of spindle orientations. In explants in which the RPE is removed, however, the proportion of vertical spindles is decreased by almost $50 \%$, suggesting that the RPE normally promotes vertical divisions.

It is unclear how the RPE influences spindle orientation in the retinal neuroepithelium, although it is clearly in an advantageous location to do it. It has been shown previously that ablation of the $\mathrm{RPE}$ in transgenic mice results in the disorganization of the normal layers in the neural retina, consistent with a role for the RPE in polarizing the developing retinal neuroepithelium (Raymond and Jackson, 1995). Moreover, mutations in the mosaic eyes gene in zebrafish, which functions in the RPE, disrupts neural retina lamination and decreases the number of Müller cells (Jensen et al., 2001), consistent with our results and the model presented in Figure 6. One possibility is that the RPE may activate an apical protein complex in some adjacent neuroepithelial cells to reorient the mitotic spindle along the basal-apical axis. Another is that the only stable state for the mitotic spindles is horizontal, and the 
RPE might, in some neuroepithelial cells, disrupt the cues associated with adherens junctions that may be required for the horizontal orientation of the spindle (Lu et al., 2001). This would allow these cells to tumble randomly away from horizontal and could explain why we do not see a bimodal distribution in the orientation of the mitotic spindles.

It is clear that the RPE is not continuously required to maintain polarity in the neonatal retinal neuroepithelium, because cells in explants of neonatal retina without the RPE maintain their preference for horizontal divisions (although fewer cells divide vertically) perhaps because adherens junctions are maintained. Moreover, the apical distribution of Numb persists for at least $3 \mathrm{~d}$ in such explants. The RPE might help initially to polarize the neural retina, which may then retain its polarization in the absence of the RPE.

Perhaps the best-studied example of how cells can influence the spindle orientation of adjacent cells is that in Caenorhabditis elegans. In the four-cell worm embryo, the $\mathrm{P}_{2}$ cell signals to the adjacent EMS cell via a Wnt signal protein, polarizing the EMS cell with respect to the site of contact with $\mathrm{P}_{2}$. This interaction reorients the mitotic spindle through $90^{\circ}$ in the EMS cell, which, as a consequence, undergoes an asymmetric division (Hyman and White, 1987; Schlesinger et al., 1999). As with other asymmetric divisions in the worm, the intracellular events that reorient the spindle may, at least in part, depend on Par proteins (Kemphues et al., 1988; Etemad-Moghadam et al., 1995; Watts et al., 1996; Hung and Kemphues, 1999; Joberty et al., 2000), which have homologs in insects and vertebrates (Izumi et al., 1998; Schober et al., 1999; Wodarz et al., 1999; Lin et al., 2000). Moreover, the mammalian RPE secretes a frizzled-related protein (SFRP-5) that acts by modulating Wnt signaling and has been suggested to play a part in polarizing retinal neuroepithelial cells (Chang et al., 1999). Thus, some of the mechanisms involved in polarizing epithelial cells may be conserved in all animals.

\section{APPENDIX}

To quantify and analyze the orientations of mitotic spindles within the three-dimensional structure of the developing retinal neuroepithelium, it was necessary to adopt an appropriate coordinate system and frame of reference. The two centrosomes of the spindle provide a convenient pair of spatial coordinates that can be related to the overall tissue orientation to indicate the spindle orientation.

The interface between the neural retina and the pigment epithelium is a convenient reference frame for the overall tissue orientation, and all spindle orientations were calculated with respect to this interface. Because the interface is not smooth, it is necessary to perform a statistical fit to allow further analysis. Theoretically, this should be of a regression plane, as shown in Figure 7. To simplify the computations, however, a regression line (rather than a regression plane) was fitted to the centermost optical section of the reconstructed volume by selecting 10-15 evenly spaced points along the RPE-retinal interface by eye, using a screen cursor. Then, all orientations were referenced to this line.

Having generated a reference axis, we then remapped the $x, y$, and $z$ coordinates of the centrosomes accordingly. To do this, the value of the intercept $c$ of the fitted regression line $(y=m x+c)$ was subtracted from all of the measured $y$ values. This is equivalent to dropping the fitted line so that its first value passes through the origin. The measured $x$ and $y$ coordinates of the centrosome pair were rotated then through an angle correspond-

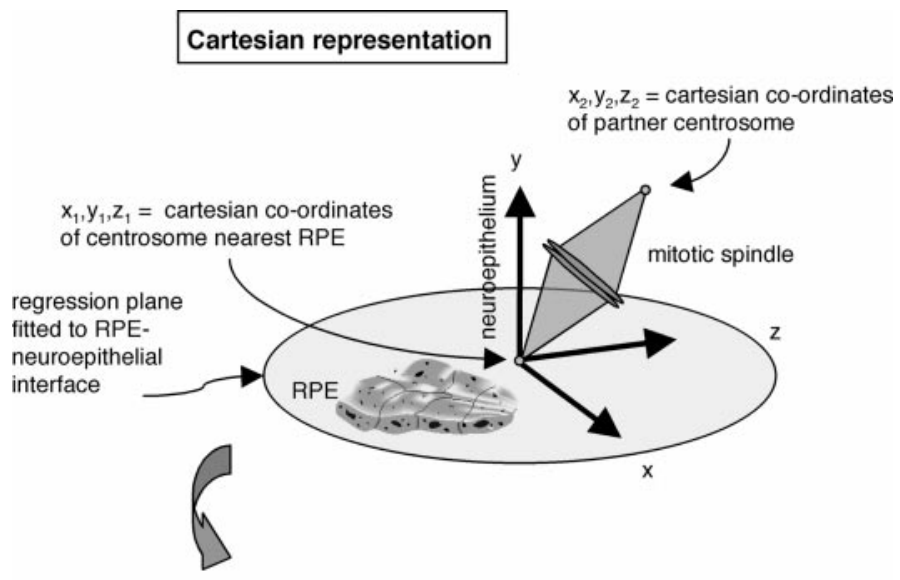

Spherical polar representation

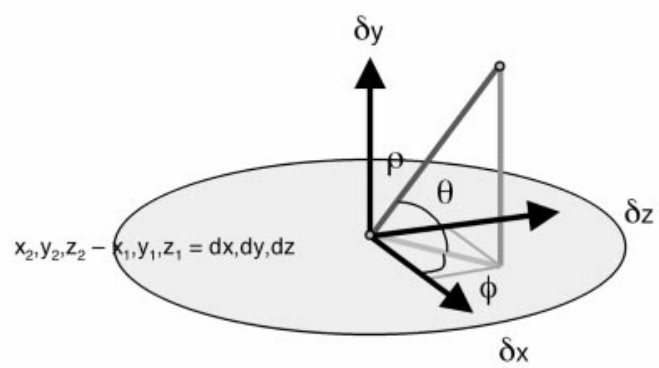

$$
\begin{array}{ll}
\rho=\sqrt{\delta x^{2}+\delta y^{2}+\delta z^{2}} & \text { intercentrosomal distance } \\
\theta=\tan ^{-1}\left(\delta y / \sqrt{\delta x^{2}+\delta z^{2}}\right) & \text { elevation above plane of epithelium } \\
\phi=\tan ^{-1}(\delta z / \delta x) & \text { rotation (azimuth) within plane of epithelium }
\end{array}
$$

Figure 7. Calculation of the orientation of mitotic spindles, using threedimensional coordinate geometry. To determine the spindle orientation, a regression fit is first made to the interface between the RPE and the neural retina to provide a reference plane or axis. The $x, y$, and $z$ Cartesian coordinates of the two centrosomes of the spindle are transformed such that they are now expressed relative to the fitted plane or line, as illustrated in the top diagram, which shows a schematic mitotic spindle. The RPE is shown directly beneath the mitotic cell in the $x-z$ plane, and the positions of the two centrosomes are indicated by the triplet coordinate pairs $x_{1}, y_{1}, z_{1}$ and $x_{2}, y_{2}, z_{2}$. To calculate the elevation of the spindle above the $x-z$ plane, it is necessary to represent this information in spherical polar coordinate form. This is done as follows: the difference between the triplets is calculated (shown in the bottom diagram) and represented by the values $\delta x, \delta y$, and $\delta z$. These values are then substituted into the equations (derived from trigonometry) shown at the bottom of the figure to yield the values $\rho$ (the distance between the centrosomes), $\theta$ (the elevation of the spindle out of the $x-z$ plane), and $\varphi$ (the rotation of the spindle about the $y$-axis, also termed the azimuth). These values are also illustrated on the right side of the bottom diagram.

ing to the slope $m$ of the fitted regression line, using the standard equations: $x=x^{\prime} \cos \phi-y^{\prime} \sin \phi$ and $y=x^{\prime} \sin \phi-y^{\prime} \cos \phi$, where $x$ and $y$ are the transformed coordinates, $\phi=\tan ^{-1} m$, and $x^{\prime}$ and $y^{\prime}$ are the raw coordinates. The $z$ values were not changed.

The $x, y$, and $z$ values corresponding to the centrosome pair were transformed then into spherical polar coordinate representation, using the equations shown in the bottom part of Figure 7. Spherical polar coordinates describe the relationship between the centrosomes of a spindle in terms of the distance between the centrosomes, the rotation within the plane of the neuroepithelium (azimuth), and the positive angle between $0^{\circ}$ (horizontal) and $90^{\circ}$ (vertical). 


\section{REFERENCES}

Carmena A, Murugasu-Oei B, Menon D, Jimenez F, Chia W (1998) Inscuteable and numb mediate asymmetric muscle progenitor cell divisions during Drosophila myogenesis. Genes Dev [Erratum (1998) 12:1241] 12:304-315.

Cepko CL (1999) The roles of intrinsic and extrinsic cues and bHLH genes in the determination of retinal cell fates. Curr Opin Neurobiol 9:37-46.

Cepko CL, Austin CP, Yang X, Alexiades M, Ezzeddine D (1996) Cell fate determination in the vertebrate retina. Proc Natl Acad Sci USA 93:589-595.

Chang JT, Esumi N, Moore K, Li Y, Zhang S, Chew C, Goodman B, Rattner A, Moody S, Stetten G, Campochiaro PA, Zack DJ (1999) Cloning and characterization of a secreted frizzled-related protein that is expressed by the retinal pigment epithelium. Hum Mol Genet 8:575-583.

Chenn A, McConnell SK (1995) Cleavage orientation and the asymmetric inheritance of Notch1 immunoreactivity in mammalian neurogenesis. Cell 82:631-641.

Etemad-Moghadam B, Guo S, Kemphues KJ (1995) Asymmetrically distributed PAR-3 protein contributes to cell polarity and spindle alignment in early C. elegans embryos. Cell 83:743-752.

Frise E, Knoblich JA, Younger-Shepherd S, Jan LY, Jan YN (1996) The Drosophila Numb protein inhibits signaling of the Notch receptor during cell-cell interaction in sensory organ lineage. Proc Natl Acad Sci USA 93:11925-11932.

Furukawa T, Mukherjee S, Bao ZZ, Morrow EM, Cepko CL (2000) rax, Hes1, and notch1 promote the formation of Muller glia by postnatal retinal progenitor cells. Neuron 26:383-394.

Gaiano N, Nye JS, Fishell G (2000) Radial glial identity is promoted by Notch1 signaling in the murine forebrain. Neuron 26:395-404

Guo M, Jan LY, Jan YN (1996) Control of daughter cell fates during asymmetric division: interaction of Numb and Notch. Neuron $17: 27-41$.

Harris WA (1997) Cellular diversification in the vertebrate retina. Curr Opin Genet Dev 7:651-658.

Horvitz HR, Herskowitz I (1992) Mechanisms of asymmetric cell division: two Bs or not two Bs, that is the question. Cell 68:237-255.

Hung TJ, Kemphues KJ (1999) PAR-6 is a conserved PDZ domaincontaining protein that colocalizes with PAR-3 in Caenorhabditis elegans embryos. Development 126:127-135.

Hyman AA, White JG (1987) Determination of cell division axes in the early embryogenesis of Caenorhabditis elegans. $\mathrm{J}$ Cell Biol $105: 2123-2135$.

Izumi Y, Hirose T, Tamai Y, Hirai S, Nagashima Y, Fujimoto T, Tabuse Y, Kemphues KJ, Ohno S (1998) An atypical PKC directly associates and colocalizes at the epithelial tight junction with ASIP, a mammalian homologue of Caenorhabditis elegans polarity protein PAR-3. J Cell Biol 143:95-106.

Jensen AM, Raff MC (1997) Continuous observation of multipotential retinal progenitor cells in clonal density culture. Dev Biol 188:267-279.

Jensen AM, Walker C, Westerfield M (2001) mosaic eyes: a zebrafish gene required in pigmented epithelium for apical localization of retinal cell division and lamination. Development 128:95-105.

Joberty G, Petersen C, Gao L, Macara IG (2000) The cell-polarity protein Par6 links Par3 and atypical protein kinase $\mathrm{C}$ to $\mathrm{Cdc} 42$. Nat Cell Biol 2:531-539.

Kaltschmidt JA, Davidson CM, Brown NH, Brand AH (2000) Rotation and asymmetry of the mitotic spindle direct asymmetric cell division in the developing central nervous system. Nat Cell Biol 2:7-12.

Kemphues KJ, Priess JR, Morton DG, Cheng NS (1988) Identification of genes required for cytoplasmic localization in early $C$. elegans embryos. Cell 52:311-320.

Knoblich JA, Jan LY, Jan YN (1995) Asymmetric segregation of Numb and Prospero during cell division. Nature 377:624-627.

Lewis J (1998) Notch signalling and the control of cell fate choices in vertebrates. Semin Cell Dev Biol 9:583-589.

Lin D, Edwards AS, Fawcett JP, Mbamalu G, Scott JD, Pawson T (2000)
A mammalian PAR-3-PAR-6 complex implicated in Cdc42/Rac1 and aPKC signalling and cell polarity. Nat Cell Biol 2:540-547.

Livesey FJ, Cepko CL (2001) Vertebrate neural cell-fate determination: lessons from the retina. Nat Rev Neurosci 2:109-118.

Lu B, Jan L, Jan YN (2000) Control of cell divisions in the nervous system: symmetry and asymmetry. Annu Rev Neurosci 23:531-556.

Lu B, Roegiers F, Jan LY, Jan YN (2001) Adherens junctions inhibit asymmetric division in the Drosophila epithelium. Nature 409:522-525. Morrison SJ, Perez SE, Qiao Z, Verdi JM, Hicks C, Weinmaster G, Anderson DJ (2000) Transient Notch activation initiates an irreversible switch from neurogenesis to gliogenesis by neural crest stem cells. Cell 101:499-510.

Raymond SM, Jackson IJ (1995) The retinal pigmented epithelium is required for development and maintenance of the mouse neural retina. Curr Biol 5:1286-1295.

Rhyu MS, Jan LY, Jan YN (1994) Asymmetric distribution of numb protein during division of the sensory organ precursor cell confers distinct fates to daughter cells. Cell 76:477-491.

Rose LS, Kemphues KJ (1998) Early patterning of the C. elegans embryo. Annu Rev Genet 32:521-545.

Schlesinger A, Shelton CA, Maloof JN, Meneghini M, Bowerman B (1999) Wnt pathway components orient a mitotic spindle in the early Caenorhabditis elegans embryo without requiring gene transcription in the responding cell. Genes Dev 13:2028-2038.

Schober M, Schaefer M, Knoblich JA (1999) Bazooka recruits Inscuteable to orient asymmetric cell divisions in Drosophila neuroblasts. Nature 402:548-551.

Spana EP, Doe CQ (1996) Numb antagonizes Notch signaling to specify sibling neuron cell fates. Neuron 17:21-26.

Spana EP, Kopczynski C, Goodman CS, Doe CQ (1995) Asymmetric localization of numb autonomously determines sibling neuron identity in the Drosophila CNS. Development 121:3489-3494.

Turner DL, Cepko CL (1987) A common progenitor for neurons and glia persists in rat retina late in development. Nature 328:131-136.

Uemura T, Shepherd S, Ackerman L, Jan LY, Jan YN (1989) numb, a gene required in determination of cell fate during sensory organ formation in Drosophila embryos. Cell 58:349-360.

Verdi JM, Schmandt R, Bashirullah A, Jacob S, Salvino R, Craig CG, Program AE, Lipshitz HD, McGlade CJ (1996) Mammalian NUMB is an evolutionarily conserved signaling adapter protein that specifies cell fate. Curr Biol 6:1134-1145.

Wakamatsu Y, Maynard TM, Jones SU, Weston JA (1999) NUMB localizes in the basal cortex of mitotic avian neuroepithelial cells and modulates neuronal differentiation by binding to NOTCH-1. Neuron 23:71-81

Wan S, Cato AM, Skaer H (2000) Multiple signalling pathways establish cell fate and cell number in Drosophila malpighian tubules. Dev Biol 217:153-165

Wang S, Barres BA (2000) Up a notch: instructing gliogenesis. Neuron 27:197-200.

Watts JL, Etemad-Moghadam B, Guo S, Boyd L, Draper BW, Mello CC, Priess JR, Kemphues KJ (1996) par-6, a gene involved in the establishment of asymmetry in early $C$. elegans embryos, mediates the asymmetric localization of PAR-3. Development 122:3133-3140.

Wodarz A, Ramrath A, Kuchinke U, Knust E (1999) Bazooka provides an apical cue for Inscuteable localization in Drosophila neuroblasts. Nature 402:544-547.

Young RW (1985) Cell differentiation in the retina of the mouse. Anat Rec 212:199-205.

Zhong W, Feder JN, Jiang MM, Jan LY, Jan YN (1996) Asymmetric localization of a mammalian numb homolog during mouse cortical neurogenesis. Neuron 17:43-53.

Zhong W, Jiang MM, Weinmaster G, Jan LY, Jan YN (1997) Differential expression of mammalian Numb, Numblike and Notch1 suggests distinct roles during mouse cortical neurogenesis. Development 124:1887-1897.

Zhong W, Jiang MM, Schonemann MD, Meneses JJ, Pedersen RA, Jan LY, Jan YN (2000) Mouse numb is an essential gene involved in cortical neurogenesis. Proc Natl Acad Sci USA 97:6844-6849. 